\title{
Blow up at the hyperbolic boundary for a $2 \times 2$ system arising from chemical engineering
}

\author{
C. Bourdarias ${ }^{*}$ M. Gisclon ${ }^{\dagger}$ and S. Junca ${ }^{\ddagger}$
}

November 22, 2009

\begin{abstract}
We consider an initial boundary value problem for a $2 \times 2$ system of conservation laws modeling heatless adsorption of a gaseous mixture with two species and instantaneous exchange kinetics, close to the system of Chromatography. In this model the velocity is not constant because the sorption effect is taken into account. Exchanging the roles of the $x, t$ variables we obtain a strictly hyperbolic system with a zero eigenvalue. Our aim is to construct a solution with a velocity which blows up at the corresponding characteristic "hyperbolic boundary" $\{t=0\}$.
\end{abstract}

AMS Classification: 35L65, 35L67, 35Q35.

Key words: hyperbolic systems, conservation laws, weak solutions, Temple systems, blow up, boundary conditions, gas chromatography, Riemann problem, Front Tracking Algorithm.

\section{Introduction}

This paper deals with the construction of a blow up solution for a one dimensional $2 \times 2$ hyperbolic system of conservation laws related to a particular isothermal gas-solid chromatography process, called "Pressure Swing Adsorption" (PSA), with two species. Each of the two species simultaneously exists under two phases: a gaseous and movable one with a common velocity $u(t, x)$ and concentration $c_{i}(t, x) \geq 0$ and a solid (adsorbed) with concentration $q_{i}, i=1,2$. One may consult [20] and [23] for a precise description of the process and [6] for a survey on various related models.

In gas chromatography, velocity variations accompany changes in gas composition, especially in the case of high concentration solute: it is known as the sorption effect. Here, the sorption effect is taken into account through a constraint on the pressure that is, in this isothermal model, on the total density:

$$
c_{1}+c_{2}=\rho(t),
$$

${ }^{*}$ Université de Savoie, LAMA, UMR CNRS 5127, 73376 Le Bourget-du-Lac, bourdarias@univ-savoie.fr

†Université de Savoie, LAMA, UMR CNRS 5127, 73376 Le Bourget-du-Lac, gisclon@univ-savoie.fr

${ }^{\ddagger}$ Université de Nice, Labo. JAD, UMR CNRS 6621, Parc Valrose, 06108 Nice, junca@unice.fr 
where the function $\rho$ is given (this is actually achieved in the experimental or industrial device). This constraint expresses that the total pressure depends only on the time. In the sequel we assume that $\rho \equiv 1$.

We assume that mass exchanges between the mobile and the stationary phases are infinitely fast (instantaneous exchange kinetics) thus the two phases are constantly at composition equilibrium: the concentrations in the solid phase are given by some relations $q_{i}=q_{i}^{*}\left(c_{1}, c_{2}\right)$ where the functions $q_{i}^{*}$ are the so-called equilibrium isotherms. A theoretical study of a model with finite exchange kinetics was presented in [2] and a numerical approach was developed in 3 .

With these assumptions, the PSA system reads:

$$
\begin{aligned}
\partial_{t}\left(c_{1}+q_{1}^{*}\left(c_{1}, c_{2}\right)\right)+\partial_{x}\left(u c_{1}\right) & =0 \\
\partial_{t}\left(c_{2}+q_{2}^{*}\left(c_{1}, c_{2}\right)\right)+\partial_{x}\left(u c_{2}\right) & =0 \\
c_{1}+c_{2} & =1 .
\end{aligned}
$$

Notice that $c_{1}, c_{2}$ must satisfy $0 \leq c_{1}, c_{2} \leq 1$.

Adding (2) and (3) we get, thanks to (4):

$$
\partial_{t}\left(q_{1}^{*}\left(c_{1}, c_{2}\right)+q_{2}^{*}\left(c_{1}, c_{2}\right)\right)+\partial_{x} u=0,
$$

i.e. the constraint (10) leads to an integral dependency of the velocity upon the concentrations. We denote $c=c_{1}$ then $c_{2}=1-c$ and the unknowns are the velocity $u$ and the concentration $c$. We write the PSA system under the form:

$$
\begin{aligned}
\partial_{x} u+\partial_{t} h(c) & =0, \\
\partial_{x}(u c)+\partial_{t} I(c) & =0,
\end{aligned}
$$

with

$$
\begin{aligned}
h(c) & =q_{1}^{*}(c, 1-c)+q_{2}^{*}(c, 1-c), \\
I(c) & =c+q_{1}^{*}(c, 1-c) .
\end{aligned}
$$

In the sequel we denote

$$
q_{1}(c)=q_{1}^{*}(c, 1-c) \quad \text { and } \quad q_{2}(c)=q_{2}^{*}(c, 1-c),
$$

thus $h=q_{1}+q_{2}$. Any equilibrium isotherm related to a given species is always non decreasing with respect to the corresponding concentration and non increasing with respect to the others (see [17]) i.e.

$$
\frac{\partial q_{i}^{*}}{\partial c_{i}} \geq 0 \text { and } \frac{\partial q_{i}^{*}}{\partial c_{j}} \leq 0 \text { for } j \neq i,
$$

thus we have immediately:

$$
q_{1}^{\prime} \geq 0 \geq q_{2}^{\prime} .
$$

PSA system (5)-(16) is completed by initial and boundary data:

$$
\left\{\begin{array}{l}
c(0, x)=c_{0}(x) \in[0,1], \quad x>0, \\
c(t, 0)=c_{b}(t) \in[0,1], \quad t>0, \\
u(t, 0)=u_{b}(t) \geq \alpha, \quad t>0, \quad \text { for some constant } \alpha>0 .
\end{array}\right.
$$


Notice that we assume in (8) an influx boundary condition, i.e. $\forall t>0, u_{b}(t)>0$ and we choose $] 0,+\infty[$ instead of $] 0,1[$ as spatial domain for the sake of simplicity.

It is well known that it is possible to analyze the system of Chromatography, and thus PSA system, in terms of hyperbolic system of P.D.E. provided to exchange the time and space variables: see 21] and also 22 for instance. In this framework, with evolution variable $x$ instead of $t$, the system is strictly hyperbolic and has a null eigenvalue. Thus $\{t=0\}$ is a characteristic boundary.

We obtained in [4, 5] an existence result for weak global entropy solutions with large $B V$ data for the concentrations and only $L^{\infty}$ data for the velocity: as in [12, 13, 19, the zero eigenvalue makes the existence possible of stratified solutions in the sense that $u(t, x)=$ $u_{b}(t) v(t, x)$ where $v$ is as regular as the concentration $c$ and more than the "initial" data $u_{b}$ (see [7]). This linearly degenerate eigenvalue makes also possible the propagation of large amplitude and high frequency waves as in 11, 10. As we will see below, we cannot expect in general to have weak global entropy solutions with $L^{\infty}$ data for the concentrations because it is possible, in that case, to build a blow up solution for some particular isotherms related for instance to ammonia or water vapor.

It is already known that systems of two hyperbolic conservation laws may blow up in the $L^{\infty}$ norm. In literature (27, 30], there are examples of blow up for one dimensional strictly hyperbolic systems of partial differential equations. For $2 \times 2$ strictly hyperbolic system there are few examples ([26]). In [31, see also [32], R. Young built a very nice example involving two Burgers equations, linearly coupled at the two boundaries. All examples occur in cases where strict hyperbolicity is lost as the solution explodes (see also H.-K.Jenssen and R. Young [16] page 630). In our example, we also loose the strict hyperbolicity of the system at the blow up point, but the blow up takes place only at the characteristic boundary $\{t=0\}$ which becomes twice characteristic and only the velocity blows up. The context of our example (gas-solid chromatography) is physically motivated and the various assumptions are physically relevant (they are achieved for particular gases) but a blow up along the $x$-axis at $t=0$ is of course artificial. Nevertheless it illustrates what may occur when $B V$ regularity is not ensured for the velocity at the physical boundary.

The aim of this paper is to present this example of blow up for a class of PSA systems. The paper is organised as follows. In Section 2, we give an overview on PSA system (5)-(6) and specify our assumptions. In Section 3 we investigate the conditions on PSA system (5) -(6) to be or not to be in the Temple class: indeed there is no blow up for such systems. In Section 4 we state, prove and comment the main result of this paper about a blow up of velocity in $L^{\infty}$.

\section{The PSA system}

\subsection{General properties}

In this section, we recall briefly some properties of PSA system (5)-(6). For more details and explanations we refer the reader to [4, 5, 6, 7].

As pointed out by Rouchon and al. ([22]), it is possible to analyse PSA system in terms of hyperbolic system of P.D.E. provided we exchange the time and space variables. In this framework the vector state will be $U=\left(\begin{array}{l}u \\ m\end{array}\right)$ where $m=u c$ is the flow rate of the first species. The first component $u$ of this vector must be understood as $u \rho$, that is the total flow 
rate. The reader should bear in mind that convexity is a relevant property of the entropy only with respect to the conservative variables $(u, m)$ (see Dafermos [14 section 4.5 page 76 or [5]). Notice that we seek for solutions satisfying $u>0$, wich ensure the system to be hyperbolic. Using the diffeomorphism $(u, c) \mapsto(u, m)$ between $\left.\mathbb{R}_{+}^{*} \times\right] 0,1\left[\right.$ and $\mathbb{R}_{+}^{*} \times \mathbb{R}_{+}^{*}$, it is more convenient to deal with the hyperbolicity, the Riemann invariants, the Riemann problem and the Front tracking algorithm with the set of variables $(u, c)$. For instance there is no partial derivative with respect to $t$ for $u$ and $c$ is a Riemann invariant.

PSA system with conservative variables $(u, m)$ takes the form

$$
\partial_{x} U+\partial_{t} \Phi(U)=0 \text { with } \Phi(U)=\left(\begin{array}{c}
h(m / u) \\
I(m / u)
\end{array}\right) .
$$

It is strictly hyperbolic with variable $x$ as the evolution variable: the two eigenvalues are 0 (linearly degenerate) and $\lambda=H(c) / u$ where

$$
H(c)=1+q_{1}^{\prime}(c)-c h^{\prime}(c)=1+(1-c) q_{1}^{\prime}(c)-c q_{2}^{\prime}(c) \geq 1 .
$$

In particular, the mathematical boundary condition is now related to the set $\{t=0\}$ and the mathematical initial data to the set $\{x=0\}$. Making use of the function $f=q_{1} c_{2}-q_{2} c_{1}$ introduced by Douglas and al. in [17, written here under the form

$$
f(c)=q_{1}(c)-c h(c),
$$

it is easy to show that the right eigenvector $r=\left(\begin{array}{c}h^{\prime}(c) \\ 1+q_{1}^{\prime}(c)\end{array}\right)$ associated to $\lambda$ satisfies $d \lambda \cdot r=\frac{H(c)}{u^{2}} f^{\prime \prime}(c)$, thus $\lambda$ is genuinely nonlinear in each domain where $f^{\prime \prime} \neq 0$. In view of the remark following (44) there is a "natural maximum principle" for the concentration $c$ :

$$
0 \leq c \leq 1
$$

and this is achieved by the solutions (see [4]).

PSA system (5)-(6) admits the two Riemann invariants $c$ and $w=\ln (u)+g(c)$ where $g$ satisfies

$$
g^{\prime}(c)=-\frac{h^{\prime}(c)}{H(c)}
$$

Indeed, for smooth solutions:

$$
\left\{\begin{aligned}
\partial_{x} c+\lambda \partial_{t} c & =0 \\
\partial_{x} w & =0 .
\end{aligned}\right.
$$

In the sequel we make use of the Riemann invariant

$$
W=u G(c)=e^{w},
$$

where

$$
G(c)=\exp (g(c)) .
$$


There are two families of smooth entropies for the PSA system (5)-(6): $u \psi(c)$ and $\phi(u G(c))$ where $\phi$ and $\psi$ are any smooth real functions, and the corresponding entropy fluxes satisfy

$$
Q^{\prime}(c)=h^{\prime}(c) \psi(c)+H(c) \psi^{\prime}(c) .
$$

The first family is degenerate convex (in variables $(u, m)$ ) provided $\psi^{\prime \prime} \geq 0$. So, we seek after weak entropy solutions which satisfy, for such functions $\psi$

$$
\partial_{x}(u \psi(c))+\partial_{t} Q(c) \leq 0
$$

in the distribution sense.

The second family is not always convex. Nevertheless, if $\pm G^{\prime \prime} \geq 0$ then $u \psi= \pm u G(c)$ is a degenerate convex entropy, with entropy flux $Q \equiv 0$, contained in the family of entropies $u \psi(c)$. So, if $G^{\prime \prime}$ keeps a constant sign on $[0,1],(c, u)$ has to satisfy, in the distribution sense:

$$
\pm \frac{\partial}{\partial x}(u G(c)) \leq 0, \quad \text { if } \pm G^{\prime \prime} \geq 0 \text { on }[0,1] .
$$

Notice that the entropies $u \psi(c)$ and the entropy $u G(c)$ are linear with respect to the velocity $u$.

\subsection{The Riemann Problem}

For the PSA system it is more convenient to begin by the resolution of the boundary Riemann problem which is characteristic. The complete Riemann problem is solved at the end of this subsection. The boundary Riemann problem has the form:

$$
\begin{aligned}
& \left\{\begin{array}{c}
\partial_{x} u+\partial_{t} h(c)=0, \\
\partial_{x}(u c)+\partial_{t} I(c)=0,
\end{array}\right. \\
& c(0, x)=c^{0} \in[0,1], \quad x>0, \quad \begin{cases}c(t, 0)=c^{+} \in[0,1], & t>0 . \\
u(t, 0)= & u^{+}>0,\end{cases}
\end{aligned}
$$

We are classically looking for a selfsimilar solution, i.e.: $c(t, x)=C(z), u(t, x)=U(z)$ with $z=\frac{t}{x}>0$ (see Fig. 1).

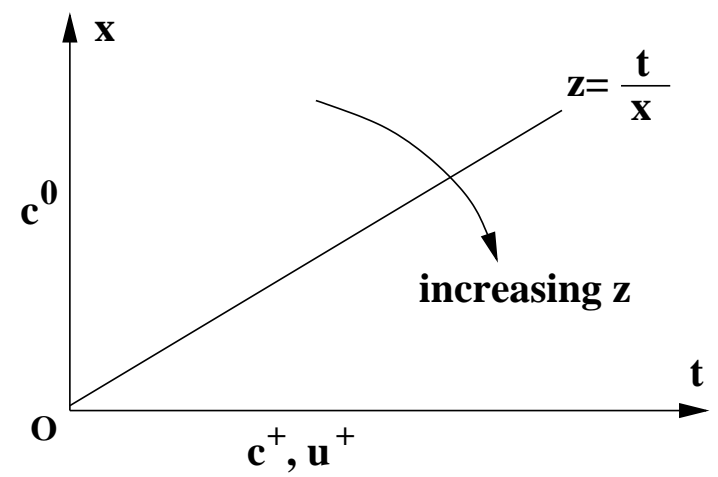

Figure 1: data for the boundary Riemann problem 
In the domain $t>0, x>0$, the boundary Riemann problem is solved with $\lambda$-waves since $\lambda$ is the only positive eigenvalue of the system. Let us recall the three following results obtained in [5], where $H$ and $f$ are given by (10, 11).

\section{Proposition 2.1 ( $\lambda$-rarefaction waves)}

Any smooth non-constant self-similar solution $(C(z), U(z))$ of (16) in an open domain $\Omega=\{0 \leq \alpha<z<\beta\}$ where $f^{\prime \prime}(C(z))$ does not vanish, satisfies:

$$
\frac{d C}{d z}=\frac{H(C)}{z f^{\prime \prime}(C)}, \quad U(z)=\frac{H(C)}{z}
$$

In particular, $\frac{d C}{d z}$ has the same sign as $f^{\prime \prime}(C)$.

Assume for instance that $0 \leq a<c^{0}<c^{+}<b \leq 1$ and $f^{\prime \prime}>0$ in $] a, b[$. Then the only smooth self-similar solution of (16) is such that:

$$
\left\{\begin{aligned}
C(z) & = & c^{0}, & 0<z<z_{0} \\
\frac{d C}{d z} & = & \frac{H(C)}{z f^{\prime \prime}(C)}, & z_{0}<z<z_{+} \\
C(z) & = & c_{+}, & z_{+}<z
\end{aligned}\right.
$$

where $z^{+}=\frac{H\left(c^{+}\right)}{u^{+}}, z^{0}=z^{+} e^{-\Phi\left(c^{+}\right)}$with $\Phi(c)=\int_{c^{0}}^{c} \frac{f^{\prime \prime}(\xi)}{H(\xi)} d \xi$. Moreover $u^{0}=\frac{H\left(c^{0}\right)}{z^{0}}$ and $U$ is given by:

$$
\left\{\begin{array}{rrrr}
U(z) & = & u_{0}, & 0<z<z_{0} \\
U(z)= & \frac{H(C(z))}{z}, & z_{0}<z<z_{+} \\
U(z)= & u_{+} & z_{+}<z
\end{array}\right.
$$

Proposition $2.2\left(\lambda\right.$-shock waves) If $\left(c^{0}, c^{+}\right)$satisfies the following admissibility condition equivalent to the Liu entropy-condition ([18]):

$$
\text { for all } c \text { between } c^{0} \text { and } c^{+}, \quad \frac{f\left(c^{+}\right)-f\left(c^{0}\right)}{c^{+}-c^{0}} \leq \frac{f(c)-f\left(c^{0}\right)}{c-c^{0}},
$$

then the Riemann problem (16) is solved by a shock wave defined as:

$$
C(z)=\left\{\begin{array}{ll}
c^{0} & \text { if } \quad 0<z<s, \\
c^{+} & \text {if } \quad s<z
\end{array}, \quad U(z)=\left\{\begin{array}{lll}
u^{0} & \text { if } & 0<z<s \\
u^{+} & \text {if } & s<z
\end{array}\right.\right.
$$

where $u^{0}$ and the speed $s$ of the shock are obtained through

$$
\frac{[f]}{u^{0}[c]}+\frac{1+h^{0}}{u^{0}}=s=\frac{[f]}{u^{+}[c]}+\frac{1+h^{+}}{u^{+}}
$$

where $[c]=c^{+}-c^{0},[f]=f^{+}-f^{0}=f\left(c^{+}\right)-f\left(c^{0}\right), h^{+}=h\left(c^{+}\right), h^{0}=h\left(c^{0}\right)$.

Proposition 2.3 ( $\lambda$-contact discontinuity ) Two states $U^{0}$ and $U^{+}$are connected by a $\lambda$-contact discontinuity with $c^{0} \neq c^{+}$if and only if $f$ is affine between $c^{0}$ and $c^{+}$. 

below.

In this paper, there is no $\lambda$-contact discontinuity since $f$ is convex: see assumption $\left(H_{3}\right)$

It appears from these results that we can build a weak entropy solution of the boundary Riemann problem (16) in a very simple way (see [5]), similar to the scalar case with flux $f$, for any data. In particular, if $f^{\prime \prime}$ has a constant sign, the boundary Riemann problem is always solved by a simple wave.

Now, for PSA system (16), we solve the Riemann problem with the following initial data:

$$
\left\{\begin{array}{l}
c(t, 0)=c^{-} \in[0,1], \\
u(t, 0)=u^{-}>0,
\end{array} \quad t<0, \quad\left\{\begin{array}{rl}
c(t, 0) & =c^{+} \in[0,1], \\
u(t, 0) & =u^{+}>0,
\end{array} \quad t>0 .\right.\right.
$$

A 0 -wave appears on the line $\{t=0\}$.

Proposition 2.4 (0-contact discontinuity ) Two distinct states $U^{-}$and $U^{0}$ are connected by a 0 -contact discontinuity if and only if $c^{-}=c^{0}$ (with of course $u^{-} \neq u^{0}$ ).

In conclusion the solution of the Rieman problem for $x>0$ and a convex function $f$ is

- $(c, u)=\left(c^{-}, u^{-}\right)$for $t<0$,

- a 0 -contact discontinuity for $t=0$,

- a $\lambda$ - wave for $t>0$,

see Fig. 2 below. In practice, since $c^{0}=c^{-}$, we first solve the boundary Riemann Problem (16), (17). Then $u^{0}$ is well defined and the 0 -contact discontinuity is automatically solved.
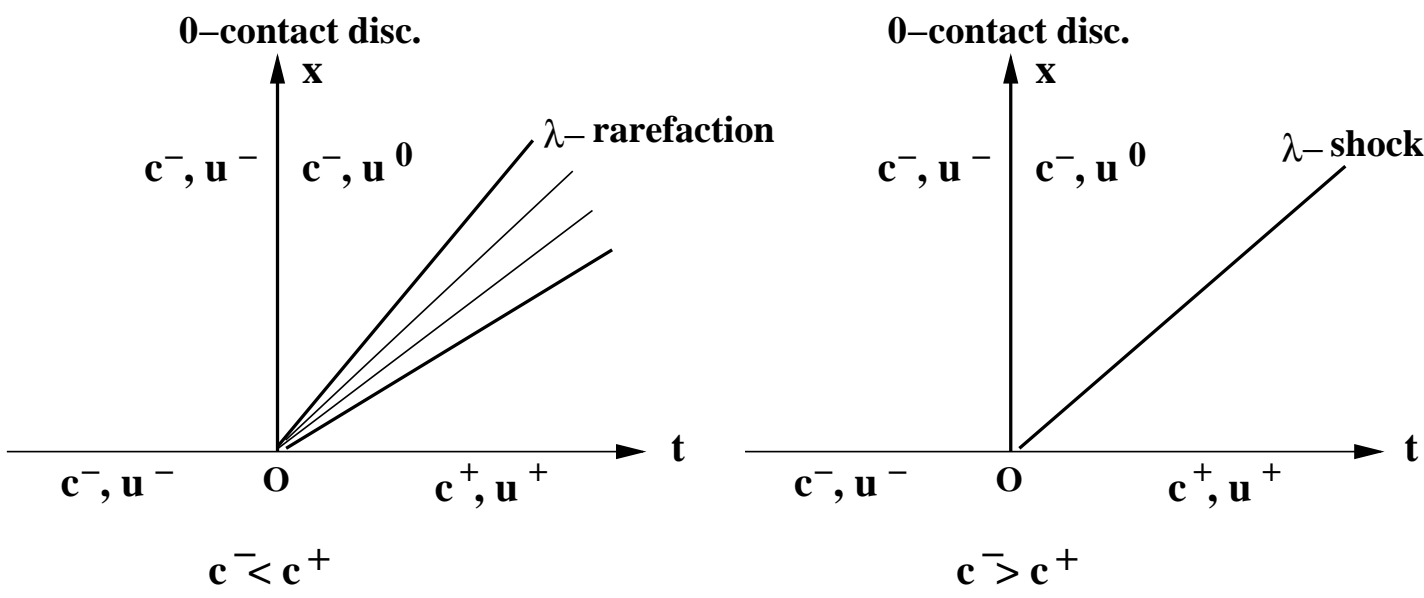

Figure 2: solution of the Riemann problem when $f^{\prime \prime}>0$.

We give also (Fig. 3) the structure of shock and rarefaction curves when the function $f$ is convex, as above, and $h^{\prime}<0$ : as stated in [7, these curves are monotonic in that case. Moreover the couple of variables $(L=\ln u, c)$ is well adapted to the study of these curves because $L+g(c)$ is a Riemann invariant. 


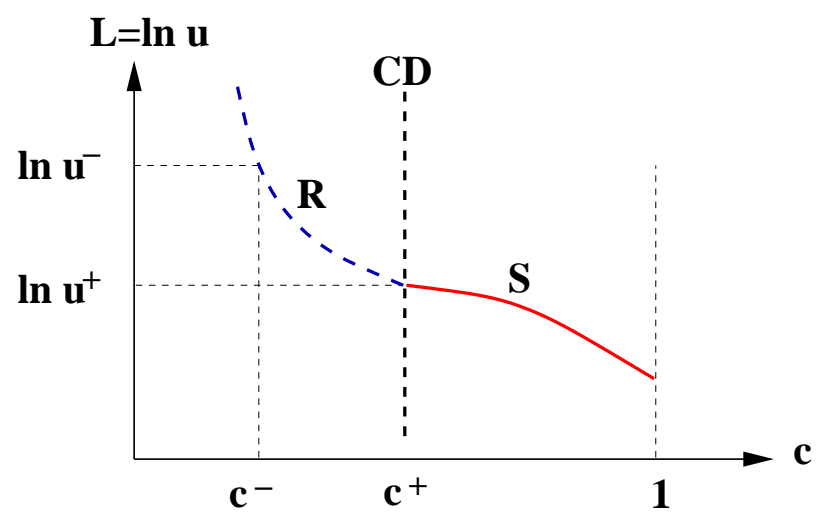

Figure 3: structure of shock curves $(\mathrm{S})$, rarefactions $(\mathrm{R})$ and contact discontinuities $(\mathrm{CD})$ in the case $f^{\prime \prime}>0>h^{\prime}$.

\subsection{Main assumptions}

PSA system has the strong property to admit a positive Riemann invariant $W=u G(c)>0$, which is also an entropy. This Riemann invariant plays a key-role in the blow up mechanism: only provided we ensure that $W$ is non decreasing with respect to $x$ we can expect, in view of (12), the velocity $u$ to increase and blow up. Indeed, if $W$ is decreasing with respect to $x$ we have $0 \leq u G(c) \leq u_{b}(t) G\left(c_{b}(t)\right)$, thus $0 \leq u \leq\left\|u_{b}\right\|_{\infty} \sup _{[0,1]} G / \inf _{[0,1]} G$ and $u$ is bounded.

Then our first assumption is:

$$
G^{\prime \prime}<0
$$

$(H 1)$ means, as seen above (15), that $-W$ is an admissible degenerate convex entropy for weak entropy solutions (with a zero entropy-flux) i.e. $\partial_{x} W \geq 0$.

In contrary, notice that in some cases, for instance an inert gas and an active gas with a Langmuir isotherm as in [4, we have $G^{\prime \prime}>0$, then $W$ is bounded and there is no blow up for the velocity in $L^{\infty}$.

The next hypothesis is useful to the construction of our blow up example in a classical hyperbolic framework of Lax with monotonic shock and rarefaction curves as in [7]:

$$
h^{\prime} \neq 0 \text {, everywhere } \quad(H 2) .
$$

Assumption (H2) means that one gas is more active than the other. Mathematically, it implies the monotonicity of $g$ and $G$ because $g^{\prime}=-h^{\prime} / H$. Thanks to $(H 2)$ rarefaction curves, which are the level line of $W$, are monotonic curves in the plane $(c, u)$.

We also assume that the eigenvalue $\lambda=H(c) / u$ is genuinely nonlinear. Our next assumption is then:

$$
f^{\prime \prime} \neq 0 \text {, everywhere } \quad(H 3) .
$$

For Temple systems (see the next section for a definition) it is well known that there is no blow up in $L^{\infty}$, then we have to investigate if the PSA system is in this class or not: 
this is done in the next section. This will lead us to state this supplementary assumption to avoid the Temple class:

$$
\text { PSA system (5)-(6) is not a Temple system (H4). }
$$

These four hypothesis are not natural, in the sense that they are not generally satisfied, but some applications are concerned, as the case of an inert gas associated with ammonia or water vapor for instance.

\section{Temple class and PSA system}

It is well known ([9, 1]) that blow up cannot occur for Temple systems with $L^{\infty}$ data. The aim of this section is thus to investigate the conditions for the PSA system (5)-(6) to be or not to be in the Temple class. Of course, in the sequel, we must avoid the Temple class to construct our blow up solution.

About Temple systems, on may consult [28, 29, 24, 15, 25. Let us recall the definition of the Temple class (29]).

Definition 3.1 (Temple class) A system of conservation laws in one space dimension is in the Temple class if it is strictly hyperbolic and satisfies the following properties:

1. there exists a system of coordinates consisting of Riemann invariants,

2. shock and rarefaction curves coincide.

For PSA system we have the following criterion:

Lemma 3.1 PSA system (5)-(6) is a Temple system if and only if $\partial_{x} W=0$ for the entropy solution of any Riemann problem (16), (21).

Proof : as seen above, PSA system is strictly hyperbolic and satisfies the first condition of the definition. There is a linearly degenerate eigenvalue (zero) and the rarefaction curves are only given by $\partial_{x} W=0$ (see (14) or [5]).

Notice that the Riemann problem for PSA system has a unique piecewise smooth entropy solution. If PSA system is in the Temple class, the second condition in the definition ensures that $\partial_{x} W=0$ everywhere. The converse is immediate.

Let us highlight a sufficient condition for the PSA system to be in the Temple class:

Proposition 3.1 If $G^{\prime \prime}=0$ then PSA system (5)-(6) is a Temple system.

Proof : according to (15), if $G^{\prime \prime} \equiv 0$ we have $\partial_{x}(u G(c))=0$ and from Lemma 3.1 PSA system is a Temple system.

We precise now some simple examples where $G^{\prime \prime}=0$.

Proposition 3.2 If the two isotherms are linear then $G^{\prime \prime}=0$.

Proof : we have $G=\exp (g), G^{\prime}=g^{\prime} \exp (g), G^{\prime \prime}=\left(g^{\prime \prime}+g^{\prime 2}\right) \exp (g)$. With $g^{\prime}=-h^{\prime} / H$, $H=1+q_{1}^{\prime}-c h^{\prime}(c)$ and $h=q_{1}+q_{2}$ we get

$$
G^{\prime \prime}=\frac{\exp (g)}{H^{2}}\left(-q_{1}^{\prime \prime}-q_{2}^{\prime \prime}-q_{2}^{\prime \prime} q_{1}^{\prime}+q_{2}^{\prime} q_{1}^{\prime \prime}\right)
$$


For two linear isotherms we have $q_{1}^{\prime \prime}=q_{2}^{\prime \prime}=0$ and then $G^{\prime \prime}=0$.

More generally we have:

Lemma 3.2 Assume (H2), then $G^{\prime \prime}=0$ if and only if there exist two real constants $\alpha, \beta$ such that

$$
\alpha q_{1}+(\alpha-1) q_{2}+c+\beta=0 \text {. }
$$

Proof : as seen above, $G^{\prime \prime}=0$ if and only if $q_{1}^{\prime \prime}+q_{2}^{\prime \prime}+q_{2}^{\prime \prime} q_{1}^{\prime}-q_{2}^{\prime} q_{1}^{\prime \prime}=0$. Setting $h_{1}=q_{1}^{\prime}, h_{2}=q_{2}^{\prime}$ this condition writes $\left(h_{1}+h_{2}\right)^{\prime}=\left(h_{1}+h_{2}\right)^{\prime} h_{2}-h_{2}^{\prime}\left(h_{1}+h_{2}\right)$ or also

$$
\frac{\left(h_{1}+h_{2}\right)^{\prime}}{\left(h_{1}+h_{2}\right)^{2}}=\frac{\left(h_{1}+h_{2}\right)^{\prime} h_{2}-h_{2}^{\prime}\left(h_{1}+h_{2}\right)}{\left(h_{1}+h_{2}\right)^{2}} \text {. }
$$

Thus $G^{\prime \prime}=0$ if and only if there exists a constant $\alpha$ such that

$$
\frac{-1}{h_{1}+h_{2}}=-\frac{h_{2}}{h_{1}+h_{2}}+\alpha,
$$

i.e. $-1=-q_{2}^{\prime}+\alpha\left(q_{1}^{\prime}+q_{2}^{\prime}\right)$, which gives immediately the condition.

In the particular case where a gas is inert, say the first one $\left(q_{1}=0\right)$, we can give a very simple criterion:

Proposition 3.3 Assume $q_{1}=0$. Then PSA system (5)-(6) is a Temple system if and only if the "active" isotherm is linear $\left(q_{2}^{\prime \prime}=0\right)$.

Proof : if $q_{2}^{\prime \prime}=0$ and $q_{1}=0$ we have $G^{\prime \prime}=0$ thanks to Proposition 3.2. Then the system is in the Temple class thanks to Proposition 3.1

Reciprocally, assume that the system is in the Temple class: following Lemma 3.1. $\partial_{x}(u G(c))=0$ holds for the entropy solution of any Riemann problem (16). Let $c_{-}, c_{+}, u_{+}$ be the data of the Riemann problem: the unknown is $u_{-}$and using the Rankine-Hugoniot conditions we have (see [5]):

$$
u_{-}=\mathcal{R}\left(c_{-}, c_{+}, u_{+}\right)=\left\{\begin{array}{cl}
u_{+} \gamma\left(c_{-}, c_{+}\right) & \text {for a shock } \\
u_{+} G\left(c_{+}\right) / G\left(c_{-}\right) & \text {for a rarefaction }
\end{array}\right.
$$

where

$$
\gamma\left(c_{-}, c_{+}\right)=\frac{\left[c+q_{1}(c)\right]-c_{+}[h]}{\left[c+q_{1}(c)\right]-c_{-}[h]}=\frac{\alpha+h_{-}}{\alpha+h_{+}}
$$

with $\alpha=\frac{[f]}{[c]}+1,[c]=c^{+}-c^{-},[f]=f^{+}-f^{-}=f\left(c^{+}\right)-f\left(c^{-}\right)$(and so on).

Since the system is in the Temple class, shock and rarefaction curves coincide, thus we have: $\frac{\alpha+h_{-}}{\alpha+h_{+}}=\frac{u_{-}}{u_{+}}=\frac{G_{+}}{G_{-}}$, then $-\alpha[G]=[h G]$. We can assume $g(0)=0$, then choosing $c_{-}=0$ and setting $c=c_{+}$, we get $[G]=G-1,[h G]=h G-K$ with $K=h(0)>0$ and $\alpha=1-h(c)$. Thus, the equality $-\alpha[G]=[h G]$, reads $G=1+K-h$.

Differentiating this relation we get $g^{\prime} G=-h^{\prime}$, then $G=\frac{-h^{\prime}}{g^{\prime}}=H$. Differentiating $G=H$ we get $g^{\prime}=\frac{H^{\prime}}{H}$, but in view of (13) we have $g^{\prime}=\frac{-h^{\prime}}{H}$ thus $h^{\prime}=-H^{\prime}=h^{\prime}+c h^{\prime \prime}$ and finally $h^{\prime \prime}=0$ i.e. $q_{2}^{\prime \prime}=0$. 
Remark 3.1 For an inert gas (for instance the first one: $q_{1}=0$ ) and an active gas with strictly convex isotherm ( $\left.q_{2}^{\prime \prime}>0\right)$, PSA system is not in the Temple class. For example, it is the case if the active gas is the ammonia or the water vapor. For other examples, see [5, 77].

It is now clear that (H4) can be satisfied in some physically relevant cases.

\section{The blow up solution}

In this section we present and prove our main result: the existence of an entropy blow up solution. It is organized as follows: first we state and comment this result, next we give a basic step allowing to increase the velocity through two non interacting waves, a shock and a rarefaction, then we repeat this process to build a piecewise Lipschitz entropy solution with growing velocity. Lastly, a sequence of such solutions leads to a blow up (in a bounded space-time domain).

\subsection{Main result}

Our main result needs four assumptions explained in subsection 2.3 .

Theorem 4.1 (Blow up for velocity) Assume (H1), (H2), (H3) and (H4). Then for any $T>0, X_{\infty}>0$, there exists a set of initial and boundary data (8) and a corresponding weak entropy solution on $[0, T] \times\left[0, X_{\infty}[\right.$ of PSA system (5)-(6) such that

$$
\|u\|_{L^{\infty}\left((0, T) \times\left(0, X_{\infty}\right)\right)}=+\infty .
$$

Actually the solution built to prove this theorem has special features.

- The velocity only blows up at the boundary $\{t=0\}$ when $x \rightarrow X_{\infty}$, with arbitrary small data.

- The system is strictly hyperbolic, but at $\left(t=0, x=X_{\infty}\right)$ it becomes degenerate hyperbolic because $\lambda=\frac{H(c)}{u}$ tends to 0 when $u$ tends to $+\infty$. Then the boundary becomes twice characteristic.

- $\forall X \in] 0, X_{\infty}\left[, u, c \in L^{\infty}(0, T ; B V(0, X)) \cap L^{\infty}(0, X ; B V(0, T))\right.$. The concentration $c$ remains bounded while $u$ blows up.

- Let $\Omega$ be a neighborhood of the critical point $\left(t=0, x=X_{\infty}\right)$ such that $\Omega \subset[0, T] \times$ $\left[0, X_{\infty}\right]$. Outside $\Omega$, we can prove that $(u, c)$ has a piecewise smooth structure, so the blow up occurs only at the boundary. Indeed, there is an accumulation of waveinteractions near $\left(t=0, x=X_{\infty}\right)$.

- To build such solutions, we have necessarily to choose the boundary concentration $c_{0}(x)=c(0, x)$ not in $B V\left(0, X_{\infty}\right)$. Else there is no blow up, see [4, 5.

\subsection{A mechanism to increase velocity}

In this section, we present the main ingredient for the construction of the blow up solution. It consists in the resolution of two consecutive boundary Riemann problems which leads to increase the velocity without increasing the concentrations. 


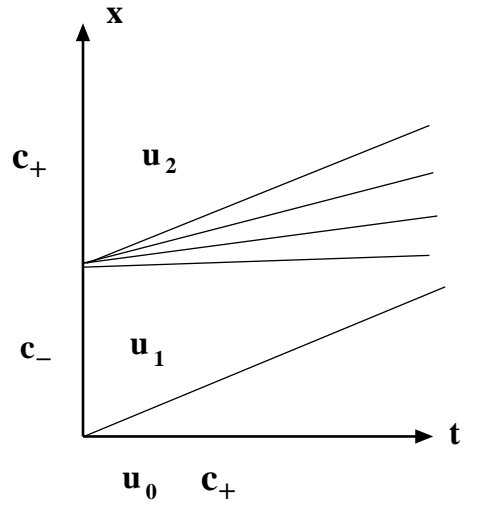

Figure 4: the two boundary Riemann problems

For the first boundary Riemann problem the data are $\left(c_{-}, c_{+}, u_{0}\right)$ chosen in such a way that the solution is a shock wave and we set (see (22) $) u_{1}=\mathcal{R}\left(c_{-}, c_{+}, u_{0}\right)$. For the second problem the data are $\left(c_{+}, c_{-}, u_{1}\right)$, the solution is necessarily a rarefaction wave and we set $u_{2}=\mathcal{R}\left(c_{+}, c_{-}, u_{1}\right)$.

The sign of $G^{\prime \prime}$ is crucial but not those of $f^{\prime \prime}$ and $h^{\prime}$ in assumptions (H3), (H2). Indeed, exchanging the labels of the two gas changes signs of $f^{\prime \prime}$ and $h^{\prime}$. In the sequel, we fix these signs:

$$
f^{\prime \prime}<0, \quad h^{\prime}<0 \quad \text { everywhere }
$$

but it is not restrictive and all variants work.

With these assumptions we must take $c_{-}<c_{+}$(see Propositions 2.2 and [4]).

We introduce now the amplification coefficient $\mathbf{R}$ defined by

$$
u_{2}=\mathbf{R} u_{0}
$$

We are going to show that this coefficient only depends upon $\left(c_{-}, c_{+}\right)$.

Lemma 4.1 The amplification coefficient satisfies $\mathbf{R}=\mathbf{R}\left(c_{-}, c_{+}\right)$.

Proof : according to (22), for the shock the Rankine-Hugoniot condition gives

$$
u_{1}=u_{0} \gamma\left(c_{-}, c_{+}\right)
$$

and for the rarefaction, we have $\partial_{x}(u G(c))=0$ then $u_{2} G\left(c_{+}\right)=u_{1} G\left(c_{-}\right)$. Finally we have

$$
u_{2}=\frac{G\left(c_{-}\right)}{G\left(c_{+}\right)} \gamma\left(c_{-}, c_{+}\right) u_{0}=\mathbf{R}\left(c_{-}, c_{+}\right) u_{0} .
$$

The following lemma justifies the label "amplification".

Lemma 4.2 We assume (H1), then the amplification coefficient satisfies $\mathbf{R} \geq 1$. 
Proof : since $G^{\prime \prime}<0$ then $\partial_{x}(u G(c)) \geq 0$ (entropy condition (15)). Through the shock wave we have $u_{1}>u_{0}, c_{-}<c_{+}$and $u_{0} G\left(c_{+}\right) \leq u_{2} G\left(c_{+}\right)$. Finally, $u_{2}=\mathbf{R} u_{0} \geq u_{0}$ then $\mathbf{R} \geq 1$.

Notice that for the shock curves we have $c_{-}<c_{+}$and $u$ is not monotonic along the process because $u_{0} \leq u_{2} \leq u_{1}$. Actually we need a strict amplification i.e. $\mathbf{R}>1$. We show that it is true for almost every choice $\left(c_{-}, c_{+}\right)$with $c_{-}<c_{+}$, provided the isotherms are analytic as we will see below. We proceed in two steps.

Lemma 4.3 $\mathbf{R}\left(c_{-}, c_{+}\right) \equiv 1$ if and only if the system is in the Temple class.

Proof : assume that $\mathbf{R}\left(c_{-}, c_{+}\right)=1$ then $u_{2}=u_{0}$. This is true for any $\left(c_{-}, c_{+}\right)$with $c_{-}<c_{+}$ and thus the shock curve connecting $\left(c_{+}, u_{0}\right)$ to $\left(c_{-}, u_{1}\right)$ and the rarefaction curve connecting $\left(c_{-}, u_{1}\right)$ to $\left(c_{+}, u_{2}=u_{0}\right)$ are the same, but traveled in opposite directions. Finally shock and rarefaction curves coincide i.e the system is in the Temple class.

Reciprocally, if the system is a Temple system we have $\partial_{x}(u G(c))=0$, then

$$
u_{0} G\left(c_{+}\right)=u_{1} G\left(c_{-}\right)=u_{2} G\left(c_{+}\right)
$$

finally $u_{0}=u_{2}$ and $\mathbf{R}\left(c_{-}, c_{+}\right)=1$.

Lemma 4.4 We assume (H1), (H3) and (H4).

If the function $\mathbf{R}$ is analytic then $\mathbf{R}\left(c_{-}, c_{+}\right)>1$ almost everywhere in the domain $c_{-}<c_{+}$.

Proof : thanks to $(H 1)$ and lemma 4.2 we have $\mathbf{R} \geq 1$. Since $\mathbf{R}$ is analytic then either $\mathbf{R}\left(c_{-}, c_{+}\right)=1$ always, either $\mathbf{R}\left(c_{-}, c_{+}\right)>1$ almost everywhere. With the assumption (H4), PSA system is not a Temple system then, thanks to the previous lemma, $\mathbf{R}>1$ almost everywhere.

Notice that the commonly used isotherms are analytic, then $\mathbf{R}$ also. With Remark 3.1 we have some relevant examples where the situation $\mathbf{R}>1$ occurs.

\subsection{A solution with growing velocity}

Following the mechanism introduced in the preceding subsection we impose constant initial data and piecewise constant concentration at the boundary such that Riemann problems at the boundary are alternatively solved by a shock or a rarefaction.

Let $N>1$ be a fixed integer, $0=x_{0}<x_{1}<\cdots<x_{2 N-1}<X=x_{2 N}, 0<\underline{c}<\bar{c}<1$ such that $\mathbf{R}(\underline{c}, \bar{c})>1$ and $u_{0}>0$. We solve PSA system (5) -(6) with the following data for $0<t<T, 0<x<X, k=0,1, \cdots, N-1$ :

$$
\left\{\begin{array}{ccc}
c(t, 0) & = & \bar{c} \\
u(t, 0) & = & u_{0}, \\
c(0, x) & =\left\{\begin{array}{ccc}
c & \text { if } & x_{2 k}<x<x_{2 k+1}, \\
\bar{c} & \text { if } & x_{2 k+1}<x<x_{2 k+2}
\end{array}\right.
\end{array}\right.
$$

With these data we prove existence and uniqueness of a piecewise Lipschitz entropy solution: 
Theorem 4.2 We assume (H1), (H2), (H3). With data (23), there exists a unique weak entropy solution in the class of piecewise Lipschitz functions. Furthermore, this solution has exactly $N$ shock curves on $[0, T] \times[0, X]$.

The uniqueness is an important feature to extend the previous entropy solution until the blow up.

Proof : to construct the solution we use the Front Tracking Algorithm as in [7] (see 8, 14 for general references). There exists a domain $Z$ (see Fig. 5), a neighborhood of the boundary $\{t=0\}$ where boundary Riemann problems do not interact. In this domain $Z$ we have an explicit solution. Let us denote by $u_{k}$ the value of $u\left(x, 0^{+}\right)$when $x_{k-1}<x<x_{k}$ for a given $k>0$.

Since $f^{\prime \prime}<0$ and $h^{\prime}<0$ we have $N$ shocks emerging from $\left(\left(x_{2 k}, t=0\right)\right)_{k=0}^{N-1}$ and $N$ rarefactions from $\left(\left(x_{2 k+1}, t=0\right)\right)_{k=0}^{N-1}$. Furthermore $u_{2 k}<u_{2 k+1}, u_{2 k}<u_{2 k+2}=\mathbf{R} u_{2 k}$ and

$$
u_{2 k}=\mathbf{R}^{k} u_{0} .
$$

Take $\delta>0$ to discretize the rarefactions as in [7] and let shocks, rarefactions and contact discontinuities interact to obtain an approximate entropy solution on $[0, T] \times[0, X]$.

As in a scalar conservation laws with piecewise constant data, no new shock can appear but shocks can disappear. It is a consequence of wave-interactions studied in [7]. We recall the following results concerning interactions:

- if a rarefaction interacts with a shock then we have a contact discontinuity and a rarefaction or a shock.

If the shock and the rarefaction have the same strength (i.e. the same absolute variation of the concentration through the simple wave) we have only a contact discontinuity;

- if two shocks interact we obtain a contact discontinuity and a stronger shock;

- if a shock interacts with a contact discontinuity, we obtain a contact discontinuity and a shock with the same strength;

- if a rarefaction interacts with a contact discontinuity, we obtain a contact discontinuity and a rarefaction with the same strength.

So, we can follow each shock, more precisely:

- if a shock disappears after an interaction with a stronger rarefaction, by convention we follow the characteristic speed associated with the eigenvalue $\lambda=H(c) / u$, and the strength of the shock is 0 ;

- if two shocks interact, they become a single shock. But, we consider this shock as two shocks to define without ambiguity the parametrization of the $N$ shocks issuing from $\left(\left(x_{2 k}, t=0\right)\right)_{k=1}^{N}$ by $N$ functions $s_{k}^{\delta}:[0, X] \rightarrow[0, T]$.

The entropy inequality $\partial_{x} u G(c) \geq 0$ yields a lower bound inf $u>0$ (see [4]): indeed we have $u G(c) \geq u_{b}(t) G\left(c_{b}(t)\right), G\left(c_{b}\right) \geq \inf _{[0,1]} G>0,0<G(c) \leq \sup _{[0,1]} G$ and $u_{b}(t) \geq \alpha>0$. Then we get an upper bound for $\lambda=H(c) / u$ : $0<\lambda<\bar{\lambda}=\frac{\sup _{[0,1]} H}{\inf u}$. Thus the speeds of the shock curves $\frac{d s_{k}^{\delta}}{d x}$ are bounded by $\bar{\lambda}$. 
Thus, we have $N$ shock curves uniformly Lipschitz. When $\delta$ goes to 0 , Ascoli's Theorem gives $N$ Lipschitz curves $\left(s_{k}\right)_{k=1}^{N}$, up to a subsequence. We denote by $U^{\delta}=\left(c^{\delta}, u^{\delta}\right)$ the approximate solution issued from the Front Tracking Algorithm with $\delta>0$ fixed. Extracting again a subsequence $\left(\delta_{n}\right)$, the sequence $U_{n}=U^{\delta_{n}}$ converges towards an entropy solution $U=(u, c)$ as in [4, 5, 7]. Let us denote by $\mathbf{S}$ the set of shocks emerging from the boundary.

$$
\mathbf{S}=\bigcup_{k=1}^{N} \Gamma_{k} \quad \text { where } \Gamma_{k}=\left\{\left(x, s_{k}(x)\right), x \in[0, X]\right\}
$$

and $\Gamma_{k}^{\delta}, S^{\delta}$ with a similar definition for the approximate solution $U^{\delta}$. Let us show that $c$ has no singularity except on $\mathbf{S}$.

Outside $\mathbf{S}$ the concentration $c$ is necessarily continuous: notice that $c$ has $B V$ regularity (see [5, 7]), then $c$ is discontinuous if and only if $c$ has shocks. Furthermore $(c, u)$ is an entropy solution hence $\partial_{t} c>0$ through the shocks. So it suffices to prove that $\partial_{t} c \leq 0$ outside $\mathbf{S}$ to conclude.

For this purpose, take any open set $\Omega$ such that $S \cap \Omega=\emptyset$. Using the uniform limit of the shock curves, we have no shock for $U_{n}$ in $\Omega$ and $n$ sufficiently large: $n>n_{1}$. So, in $\Omega$, any jump of $c$ satisfies $\left[c^{\delta_{n}}\right] \leq 0$ since it is in a rarefaction domain $\Omega$ for $n>n_{1}$. That is to say $\partial_{t} c^{\delta_{n}} \leq 0$ in $\Omega$ for $n>n_{1}$. Passing to the limit we get $\partial_{t} c \leq 0$. Then, $c$ is continuous in $\Omega$. This is true for any open set outside $\mathbf{S}$, so $c$ is continuous except on $\mathbf{S}$.

Since $c$ is continuous outside a finite number of shocks, we can obtain $c$ and $u$ by the method of characteristics, as in [7. More precisely, it was proved in [7] that any entropy solution with a continuous concentration is unique and can be constructed by the method of characteristics. Since $\lambda$ is bounded, the characteristics are Lipschitz continuous, so $c$ is Lipschitz too in the complementary of $\mathbf{S}$, which prove the piecewise Lipschitz structure of the concentration for the entropy solution. Notice that $u$ is less regular than $c$ because there are contact discontinuities emerging at each interaction between two waves.

Uniqueness: the piecewise Lipschitz structure allows us to prove uniqueness. It is a simple extension of a theorem proved in 4 for piecewise smooth solutions. For completeness, we give the key points of this proof. Under the first shock, namely in the domain $Z_{0}$ (see Fig. 5), $(u, c)$ is unique and determined by the method of characteristics. In our case, $(u, c)$ is constant in $Z_{0}$, but we do not use this fact to give a general argument and to use it for the other domains.

Over the first shock and under the second one, namely domain $Z_{1}$, the characteristics are well defined if we know $u$ on the upper side of $\Gamma_{1}$ since $\partial_{x} u G(c)=0$ on $Z_{1}$. Indeed we have the following equations in variables $(u, c)$ for some functions $\mathcal{F}$ and $\mathcal{S}$ :

$$
\begin{aligned}
& \partial_{x} W \quad=\quad 0, \quad \text { in } Z_{0} \cup Z_{1} \text {, } \\
& \partial_{t} c+W \phi(c) \partial_{x} c=0, \quad \text { in } Z_{0} \cup Z_{1} \text {, } \\
& \partial_{t} \chi(t, s, x) \quad=\quad W \phi(c), \quad \text { in } Z_{0} \cup Z_{1} \text {, } \\
& W_{1}-W_{0} \quad=\mathcal{F}\left(c_{0}, c_{1}\right), \quad \text { on } \Gamma_{1} \text {, } \\
& \frac{d s_{1}}{d t} \quad=\mathcal{S}\left(W_{0}, c_{0}, c_{1}\right), \quad \text { on } \Gamma_{1}
\end{aligned}
$$

with $W(t, 0), c(t, 0), c(0, x)$ are given functions and $\phi(c)=1 /(H(c) G(c))$.

The trick in [4] is to work in variable $t$. In the same way, $t$ is an admissible parameter for the shock curves $\Gamma_{k}$ : $\frac{d s_{k}}{d t}$ is well defined since we have BV estimates for $\ln u$, thus $\lambda$ 
avoids zero. Moreover $W$ has jumps with respect to $t$ (contact discontinuities for $u$ ) and the product $\frac{1}{W \phi(c)} \partial_{t} c$ may not be defined.

The first two equations are equations for the Riemann invariants $W$ and $c$. The third equation is the equation of characteristics associated to the eigenvalue $\lambda$ where $\chi(t, t, x)=x$. The fourth equation is the Rankine-Hugoniot equation, where $c_{i}$ and $W_{i}$ are the traces of $c$ in $Z_{i}$ on $\Gamma_{1}$. This relation gives us $W$ in $Z_{1}$ if we know $\Gamma_{1}, c_{0}$ and $c_{1}$. Indeed $\Gamma_{1}$ is an unknown curve, but the last equation determines it with an ordinary differential equation.

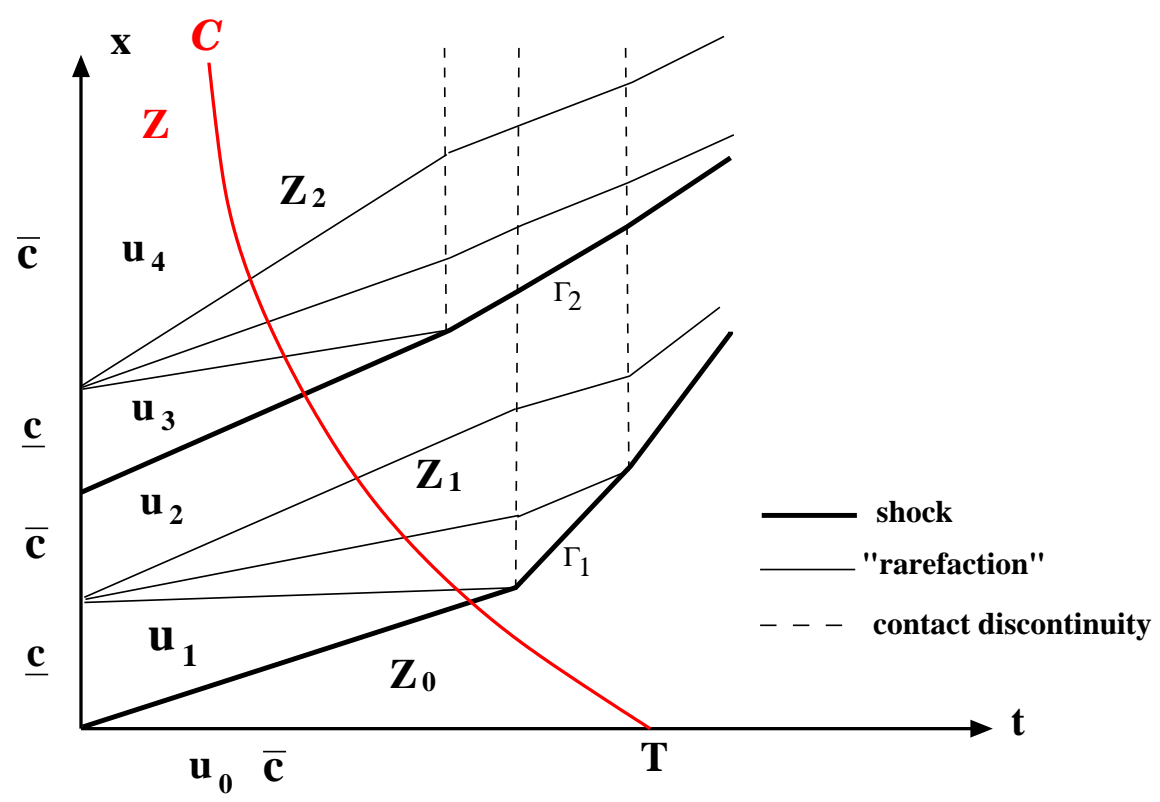

Figure 5: first interactions and free domain $\mathrm{Z}$

At $t=0$, we know $W_{0}, c_{0}, c_{1}$ so we know $\Gamma_{1}$ and $W_{1}$ locally near the corner for all $t<\tau$, and then for all $x$. We use a local uniqueness theorem as the Cauchy-Lipschitz Theorem. Notice that when we know $W_{1}$ for $t<\tau$, the characteristics are also defined for all $x$ thanks to $\partial_{x} W=0$. So, we have a uniqueness result in $Z_{0} \cup \Gamma_{1} \cup Z_{2}$ for $t<\tau$ and we extend the uniqueness result until $t=T$. Next, we use the same technique for the second shock curve and so on.

Now, as in [4], $U=(c, u)$ is unique. So, the whole sequence $U^{\delta}$ tends to $U$.

\subsection{Blow up of the velocity}

Now, we use data (23) with $N=+\infty$ i.e. $\left(x_{k}\right)_{k \in \mathbb{N}}$ is an increasing sequence such that $\lim _{k \rightarrow+\infty} x_{k}=X_{\infty}$. Then, the concentration remains bounded at the boundary but the $B V$ norm of $c$ and the $L^{\infty}$ norm of $u$ blow up at the boundary $t=0$. Near $\left(t=0, x=X_{\infty}\right)$, there is an accumulation of oscillations for the concentration and an accumulation of interactions between shocks and rarefactions.

Let $Z$ be the subset of $[0, T] \times\left[0, X_{\infty}\right.$ [ defined in the previous subsection: it is a neighborhood 
of the vertical segment $\{0\} \times\left[0, X_{\infty}\right.$ [. Indeed, the first interaction takes place at $\left(t^{1}, x^{1}\right)$ : since a contact discontinuity propagates vertically, we have first to cut the set $\left\{t>t^{1}, x>x^{1}\right\}$. We do the same work, for all first interactions of the $2 N$ Riemann problems issuing from the boundary.

Then $Z=\left\{0<x<X_{\infty}, \quad 0<t<z(x)\right\}$ where the function $z:\left[0, X_{\infty}\right] \rightarrow[0, T]$ is piecewise constant on $[0, X]$ for any $X<X_{\infty}$, non increasing, positive before $X_{\infty}, z(0)=T$, $z\left(X_{\infty}\right)=0$.

On $Z$, we exactly know the solution and

$$
\lim _{(t, x) \in Z, x \rightarrow X_{\infty}} u(t, x)=+\infty .
$$

Proof of Theorem 4.1; let $0<X<X_{\infty}$. By previous construction, we get a unique piecewise smooth entropy solution $U^{X}$ on $[0, T] \times[0, X]$. If $0<X<Y<X_{\infty}$, we get $U^{Y}$ and by uniqueness, $U^{X}=U^{Y}$ on $[0, T] \times[0, X]$. So, as for an ordinary differential equation, we can consider the unique maximal solution on $[0, T] \times\left[0, X_{\infty}[\right.$. Before interaction between the solutions of the Riemann problems issuing from the boundary, we know explicitly $U$ in $Z$. From geometric increasing of $u(0, x)$ when $x \rightarrow X_{\infty}$, we get a blow up for $u$ at $t=0$, $x=X_{\infty}$.

Furthermore, the characteristic slope $1 / \lambda$ goes to infinity near $\{0\} \times\left\{X_{\infty}\right\}$ but outside a suitable "triangular neighborhood" of this point we get a determination domain where the solution has BV regularity and there is no blow up. This determination domain has the form

$$
\mathcal{T}=\left\{(t, x) ; 0<t<T, 0<x<\min \left(X+\frac{t}{\bar{\lambda}}, X_{\infty}\right\}\right.
$$

for any $X$ in $] 0, X_{\infty}[$.

\section{Conclusion}

To conclude this paper, let us give some comments about this topic.

- It is a new physical example of hyperbolic partial differential equations exhibiting a blow up. However, this chemical model becomes not physically relevant for too large velocity, $u>>1$.

- It follows from our blow up example that general $L^{\infty}$ theory for PSA system only with $c_{0}, c_{b}, \ln u_{b} \in L^{\infty}$ is impossible. But, since our example does not blow up at finite distance of the boundary, a natural conjecture is: "if $\ln u$ remains bounded at the boundary $t=0$, there is no blow up".

- We also conjecture that our solution can be defined for $x \geq X_{\infty}$ as an entropy locally piecewise smooth solution outside the boundary, with infinite velocity on $\{0\} \times$ $\left[X_{\infty},+\infty[\right.$.

Finally we give some comparisons with other blow up solutions for $2 \times 2$ systems. The reader can also consult [27] and the memoir [26].

- There is a very simple and impressive example in [31, 32, with two Burgers equations linearly coupled on two boundaries. This solution blows up in finite time $T$ for all $x$. In contrary, for our example, there is only one boundary and the solution only blows up on this boundary. 
- As in 16, it is an example exhibiting a blow up of amplitude where at the same time the system looses strict hyperbolicity. This is a special feature of $2 \times 2$ systems, since for larger systems, at least $3 \times 3$ systems, this is well known that blow up can occur without loosing the strict hyperbolicity.

\section{References}

[1] S. Bianchini. Stability of $L^{\infty}$ solutions for hyperbolic systems with coinciding shocks and rarefactions. SIAM J. Math. Anal., 33(4):959-981, 2001.

[2] C. Bourdarias. Sur un système d'edp modélisant un processus d'adsorption isotherme d'un mélange gazeux. (french) [on a system of p.d.e. modelling heatless adsorption of a gaseous mixture]. M2AN, 26(7):867-892, 1992.

[3] C. Bourdarias. Approximation of the solution to a system modeling heatless adsorption of gases. SIAM J. Numer. Anal., 35(1):13-30, 1998.

[4] C. Bourdarias, M. Gisclon, and S. Junca. Some mathematical results on a system of transport equations with an algebraic constraint describing fixed-bed adsorption of gases. J. Math. Anal. Appl., 313(2):551-571, 2006.

[5] C. Bourdarias, M. Gisclon, and S. Junca. Existence of weak entropy solutions for gas chromatography system with one or two actives species and non convex isotherms. Commun. Math. Sci., 5(1):67-84, 2007.

[6] C. Bourdarias, M. Gisclon, and S. Junca. Hyperbolic models in gas-solid chromatography. Bol. Soc. Esp. Mat. Apl., 43:29-57, 2008.

[7] C. Bourdarias, M. Gisclon, and S. Junca. Strong stability with respect to weak limit for a hyperbolic system arising from gas chromatography. arXiv:0907.1733v2. Submitted in Methods and Applications of Analysis.

[8] A. Bressan. Hyperbolic Systems of Conservation Laws. The One-Dimensional Cauchy Problem, volume 20 of Oxford Lecture Series in Mathematics and its Applications. Oxford University Press, 2008.

[9] A. Bressan and P. Goatin. Stability of $L^{\infty}$ solutions of Temple class systems. Differential Integral Equation, 13(10-12):1503-1528, 2000.

[10] C. Cheverry, O. Guès, and G. Métivier. Oscillations fortes sur un champ linéairement dégénéré. (french) [strong oscillations on a linearly degenerate field]. Ann. Sci. Ecole Norm. Sup., (4) 36(5):691-745, 2003.

[11] C. Cheverry, O. Guès, and G. Métivier. Large-amplitude high-frequency waves for quasilinear hyperbolic systems. Adv. Differential Equations, 9(7-8):829-890, 2004.

[12] A. Corli and O. Guès. Local existence of stratified solutions to systems of balance laws. In Ann. Univ. Ferrara Sez., volume VII, pages 109-119, Workshop on Partial Differential Equations, Ferrara, 1999.

[13] A. Corli and O. Guès. Stratified solutions for systems of conservation laws. Trans. Amer. Math. Soc., 353(6):2459-2486, 2001.

[14] C. Dafermos. Hyperbolic Conservation Laws in Continuum physics. Springer, Heidelberg, 2000. 
[15] A. Heibig. Existence and uniqueness of solutions for some hyperbolic systems of conservation laws. Arch. Ration. Mech. Anal., 126:79-101, 1994.

[16] H.-K. Jenssen and R. Young. Gradient driven and singular flux blowup of smooth solutions to hyperbolic systems of conservation laws. J. Hyperbolic Differ. Equ., 1(4):627$641,2004$.

[17] M. Douglas Levan, C.A. Costa, A.E. Rodrigues, A. Bossy, and D. Tondeur. Fixed-bed adsorption of gases: Effect of velocity variations on transition types. AIChE Journal, 34(6):996-1005, 1988.

[18] T.P. Liu. The entropy condition and the admissibility of shocks. J. Math. Anal. Appl., 53:78-88, 1976.

[19] A. Museux. Stratified weak solutions of the 1-d lagrangian euler equations are viscosity solutions. Adv. Differential Equations, 9(11-12):1395-1436, 2004.

[20] H. K. Rhee, R. Aris, and N.R. Amundson. On the theory of multicomponent chromatography. Philos. Trans. R. Soc. Lond., (267):419-455, 1970.

[21] H. K. Rhee, R. Aris, and N.R. Amundson. First-Order Partial Differential Equations, volume I. Prentice-Hall, 1986.

[22] P. Rouchon, M. Sghoener, P. Valentin, and G. Guiochon. Numerical simulation of band propagation in nonlinear chromatography. In Chromatographic Science Series, volume 46, New York, 1988. Eli Grushka, Marcel Dekker Inc.

[23] P.M. Ruthwen. Principles of adsorption and adsorption processes. Wiley Interscience, 1984.

[24] D. Serre. Solutions à variations bornées pour certains systèmes hyperboliques de lois de conservation. J. Differential Equations, 68:137-168, 1987.

[25] D. Serre. Systèmes de lois de conservation I. Diderot, Paris, 1996.

[26] M. Sever. Distribution solutions of nonlinear Systems of conservation Laws. Memoirs of the AMS, 2007.

[27] W. Szeliga and R. Young. Blowup with small bv data in hyperbolic conservation laws. Arch. Ration. Mech. Anal., 179:31-54, 2005.

[28] B. Temple. Systems of conservation laws with coinciding shock and rarefaction curves. In J. Smoller, editor, Nonlinear Partial Differential Equations, volume 17, pages 143151, Providence, RI, 1983. Amer. Math. Soc.

[29] B. Temple. Systems of conservation laws with invariant manifolds. Trans. Amer. Math. Soc., 280:781-795, 1983.

[30] R. Young. Exact solutions to degenerate conservation laws. SIAM J. Math. Anal., 1999.

[31] R. Young. Blowup in hyperbolic conservation laws. Contemporary Mathematics, 1(2):269-292, 2003.

[32] R. Young. Blowup of solutions and boundary instabilities in nonlinear hyperbolic equations. Commun. Math. Sci., 1(2):269-292, 2003. 